\title{
Silencing of indoleamine 2,3-dioxygenase enhances dendritic cell immunogenicity and antitumour immunity in cancer patients
}

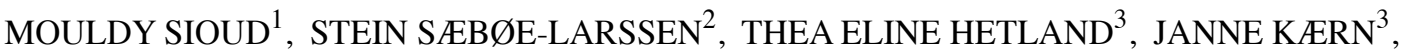 \\ ANNE MOBERGSLIEN $^{1}$ and GUNNAR KVALHEIM ${ }^{2}$ \\ Departments of ${ }^{1}$ Immunology, ${ }^{2}$ Cell Therapy and ${ }^{3}$ Gynecology, \\ Oslo University Radium Hospital, Montebello, N-0310 Oslo, Norway
}

Received January 2, 2013; Accepted February 20, 2013

DOI: $10.3892 /$ ijo.2013.1922

\begin{abstract}
Dendritic cells (DCs) are being explored as a therapeutic vaccine for cancers. However, their immunogenic potential is limited by the presence of immunosuppressive factors. Among these factors is the tryptophan-degrading enzyme indoleamine 2,3-dioxygenase (IDO). In this study, we have investigated the safety, immunogenicity and clinical response of IDO-silenced DC vaccine in four patients with gynecological cancers. DCs were transfected with IDO small interfering RNA and mRNA encoding human telomerase reverse transcriptase (hTERT) or survivin, two universal tumour antigens. Silencing of IDO in DCs did not affect the expression of the co-stimulatory molecules CD80 and CD86, but enhanced the expression of the CCR7 and CD40 molecules. IDO-silenced DCs showed superior potency to activate allogeneic $\mathrm{T}$ cells compared to their IDO-positive counterparts. The immunisation with this novel DC cancer vaccine was well tolerated and all patients developed delayed-type hypersensitivity skin reaction and specific T-cell response against hTERT and survivin tumour antigens. Perhaps most importantly, the immune response seen in the patients was related to objective clinical response. Thus, IDO silencing can enhance the immunogenic function of DCs in vitro and in vivo. Overall, the data provide proof-of-principle that immunisation with IDO-silenced DC vaccine is safe and effective in inducing antitumour immunity.
\end{abstract}

\section{Introduction}

Since their discovery in 1973 by Steinman, dendritic cells (DCs) have gained the attention of immunologists because they are extremely efficient in activating $\mathrm{T}$ cells $(1,2)$. On interaction with $\mathrm{DCs}$, naïve $\mathrm{CD} 4^{+} \mathrm{T}$ cells and $\mathrm{CD} 8^{+} \mathrm{T}$ cells

Correspondence to: Dr Mouldy Sioud, Department of Immunology, Oslo University Radium Hospital, Montebello, N-0310 Oslo, Norway E-mail: mosioud@medisin.uio.no

Key words: siRNA, dendritic cells, cancer vaccine, indoleamine 2,3-dioxygenase can differentiate into antigen-specific effector $\mathrm{T}$ cells with different functions (2). DCs can also interact with cells of the innate immune system, including natural killer cells, phagocytes and mast cells $(1,2)$. Moreover, they have an important role in controlling humoral immunity by directly interacting with B cells $(3,4)$. In addition to immune activation, DCs are also involved in immune suppression (5). These two opposite functions are important for the normal function of immune system. However, with respect to cancer immunotherapy, counter-regulatory responses antagonise the ability to induce robust and sustained immune responses that can eventually lead to tumour regression. In the case of therapeutic vaccines, immunity must be effectively induced when disease is already firmly established (6-9).

For clinically effective immune responses against tumours, there is a need to inhibit the expression of the factors that dampen immune responses in patients. This could potentially lead to longer activation of DCs, T and NK cells, and might be beneficial for cancer immunotherapy. Among the immune inhibitory molecules expressed by DCs is indoleamine 2,3-dioxygenase (IDO), an enzyme that conveys immunosuppressive effects by degrading tryptophan, an essential amino acid required for T-cell proliferation and survival (10-12). Depletion of tryptophan by IDO in the local microenvironment induces T-cell apoptosis and impairs normal T-cell function (13). IDO-expressing DCs can also induce differentiation of naïve $\mathrm{CD} 4^{+} \mathrm{T}$ cells into regulatory $\mathrm{T}$ cells ( $\mathrm{T}$-regs) that further suppress antitumor immunity (14). Moreover, various tryptophan metabolites such as $\mathrm{N}$-formylkynurenine and quinolinate are directly immuno-suppressive of T-cell activation $(10,11)$. These findings indicate that the regulation of tryptophan metabolism by IDO in DCs is a highly adaptable modulator of immunity. Indeed, injection of IDO-positive DCs into mice suppressed the activation of antigen-specific $\mathrm{T}$ cells in the lymph nodes draining the injection site (10). Effector T cells starved of tryptophan were unable to proliferate and enter into G1 cell cycle arrest. Interestingly, in vitro studies have shown that IDO-positive DCs were susceptible to killing by naturally occurring IDO-reactive cytotoxic T cells (15).

Based on the findings indicated above, a promising strategy for enhancing immunity in patients with cancer would be the blockade of IDO expression in DCs. To this end, we have 
Table I. Patient characteristics.

\begin{tabular}{|c|c|c|c|c|c|}
\hline $\begin{array}{l}\text { Patient } \\
\text { identity }\end{array}$ & Age & Stage/subtype ${ }^{a}$ & $\begin{array}{l}\text { Time at } \\
\text { diagnosis }\end{array}$ & $\begin{array}{c}\text { Time to } \\
\text { progression }\end{array}$ & $\begin{array}{l}\text { Chemotherapy prior to } \\
\text { IDO-silenced DC vaccine }\end{array}$ \\
\hline CU-1 & 56 & $\begin{array}{l}\text { Cancer } \\
\text { Ovarii stage III C }\end{array}$ & June 2006 & February 2008 & 4 different chemotherapy combinations \\
\hline CU-2 & 58 & $\begin{array}{l}\text { Cancer } \\
\text { Corporis uteri c.met }\end{array}$ & July 2004 & March 2010 & 1 chemotherapy combination \\
\hline CU-3 & 45 & $\begin{array}{l}\text { Cancer } \\
\text { Ovarii stage III C }\end{array}$ & September 2007 & September 2007 & 4 different chemotherapy combinations \\
\hline CU-4 & 57 & Cancer Peritonei & January 2009 & September 2010 & 2 different chemotherapy combinations \\
\hline
\end{tabular}

${ }^{\mathrm{a} A l l}$ three cancer types are usually referred as ovarian cancer and patients are given the same therapies.

developed several effective small interfering RNA (siRNAs) targeting IDO that exhibited a silencing potency at nanomolar concentrations (16). Importantly, IDO gene silencing enhanced the ability of DCs to stimulate T cells in vitro (16). In the present study, we sought to assess the feasibility, safety and immunogenicity of IDO-silenced DC cancer vaccine in patients with gynecological cancers such as ovarian cancer. Epithelial ovarian cancer is the leading cause of death from gynecologic malignancies (17). Despite an initial response rate of 65 to $80 \%$ to frontline therapy, recurrence arises in the majority of cases, leading a relative 5-year survival rate of only $47 \%$. Hence, this situation highlights the need of new optimized therapeutic strategies. Collectively, the data indicate that IDO-silenced DC vaccine is safe and effective in inducing antitumour immunity. Indeed, all four vaccinated patients developed immune response to the vaccine. The ability to enhance the immunostimulatory potency of DCs by silencing IDO expression would benefit patients with cancers.

\section{Materials and methods}

Patients. The patient characteristics are summarized in Table I. Two patients with cancer ovarii stage III C, one patient with metastatic cancer corporis uteri, and one patient with cancer peritonei were included in the study. These cancer types are usually considered as ovarian cancer. All patients had recurrent disease and had received multiple chemotherapy treatments when they were enrolled in the IDO-silenced DC vaccine. Prior to being included in the study, patients were informed of the investigative nature of this study, and written consent in accordance with institutional regulation was obtained prior to study entry. The use of the IDO-silenced DC vaccine as compassionate use was approved by Norwegian Medicine Agency and conducted in accordance to the provisions of the Declaration of Helsinki.

Generation of DCs for vaccination. Autologous DC vaccines were produced under standard Good Manufacturing Practice (GMP) conditions as described elsewhere $(18,19)$. Fast DCs were generated from monocytes as described by Tanaka et al (20). In brief, monocyte-rich adherent cell fractions from leukapheresis products were collected and cultured for two days in CellGro GMP DC medium supplemented with GM-CSF and IL-4 and then matured for one day by adding IL-1, IL-6, TNF- $\alpha$ and PGE-2. The resulting fast DCs $\left(5 \times 10^{7}\right.$ cells/cuvette) were transfected with either hTERT or suvivin mRNA $(10 \mu \mathrm{g})$ in combination with IDO siRNA (15-20 $\mu \mathrm{g})$ by square wave electroporation as described elsewhere (18). After transfection, the cells were cultured for one night prior to cryopreservation into separate vaccine doses $\left(5 \times 10^{6}\right.$ cells $\left./ 0.5 \mathrm{ml}\right)$.

Tumour antigens and siRNAs. mRNAs encoding hTERT and survivin proteins, two universal tumour antigens $(21,22)$, were produced as described previously under standard GMP conditions (18). The synthesised mRNAs were 5'-capped in order to be translated. With respect to siRNA, the following set of DNA oligonucleotides (MWG, Ebersberg, Germany) were used for in vitro transcription. Underlined letters indicate siRNA sense and antisense transcripts. Sense strand: oligo 1, 5'-CGTTTAATACGACTCACTATAGGTCCGTGAGTTTG TCCTTTCAA-3'; oligo 2, 5'-TTGAAAGGACAAACTCACGG ACTATAGTGAGTCGTATTAAACG-3'. Antisense strand: oligo 1,5'-CGTTTAATACGACTCACTATAGAAAGGACAA ACTCACGGACTG-3'; oligo 2, 5'-CAGTCCGTGAGTTTGTC CTTTCTATAGTGAGTCGTATTAAACG-3'. Prior to transcription, the DNA oligonucleotides were annealed to form double stranded DNA and siRNA sense and antisense strands were transcribed from the double-stranded DNA template (40 $\mu \mathrm{g} / 1 \mathrm{ml}$ reaction) using Ribomax large scale RNA production system-7 as described by the manufacturer's instructions (Promega, Madison, WI, USA). The transcripts were purified under standard GMP conditions (18). The in vitro transcribed RNA strands have the following sequence: sense strand, 5'-GGUCCGUGAGUUUGUCCUUUCAA-3', antisense strand 5'-GAAAGGACAAACUCACGGACUG-3'.

Vaccination. IDO-silenced DC vaccines were described as indicated above. The four patients received a combination of hTERT (right arm) and survivin (left arm) DC vaccines supplemented with IDO siRNA. Patients were revaccinated weekly for 4 weeks after the first DC injection. Booster vaccinations as a follow-up treatment were performed each 4 weeks. At each vaccination visit, a comprehensive assessment of adverse drug reactions, blood screening, physical examination and assessment of ECOG performance status 
(PS) was performed as described previously (19). PBMCs were isolated from patient peripheral blood using density centrifugation (Lymphoprep; Nycomed, Oslo, Norway), washed and frozen in RPMI supplemented with 20\% FCS and $10 \%$ DMSO until use. Microbiologic and endotoxin tests of DCs, mRNA and siRNA preparations were performed before use and were negative at all times.

Delayed-type hypersensitivity. Delayed-type hypersensitivity (DTH) skin tests were performed at visit one (baseline) and at all the following weekly vaccination visits from week 2 and onwards. A positive skin reaction was defined as erythema and induration of $>5 \mathrm{~mm}$ diameter $48 \mathrm{~h}$ after vaccine injection.

Allogeneic lymphocyte reactions (MLRs). Allogeneic $\mathrm{CD}^{+}$ and $\mathrm{CD}^{+} \mathrm{T}$ cells were isolated from Buffy coats by direct magnetic labelling with the appropriate microbeads according to the manufacturer's instructions (Invitrogen Dynal AS, Oslo, Norway). Co-cultures were performed as previously described (23). After 5 days in culture, proliferation was analysed by $\left[{ }^{3} \mathrm{H}\right]$-thymidine incorporation. The expression of the activation markers presented in this study was performed after DC vaccine thawing and further culturing overnight.

Recombinant hTERT and survivin proteins. A recombinant hTERT sequence encoding amino acids 563-735 of the hTERT protein was cloned into the NdeI site of the Pet- $28 \mathrm{~b}(+)$ vector (Stratagene, La Jolla, CA, USA) in frame with the N-terminal 6X His tag/thrombin sequence. The protein was produced in E. Coli BL21 codon Plus (DE3)-RIPL (Stratagene) and purified by NiNTA chromatography under denaturing conditions. All fractions were tested by western blot analysis with anti-His tag antibodies and the fractions of interest were dialyzed against PBS buffer and sterile filtered before use. Recombinant human survivin protein was purchased from BioVision Inc (Milpitas, CA, USA).

Monitoring of T-cell responses. Prior to vaccination and at week 9-10, T-cell responses were assessed in vitro against recombinant hTERT and survivin proteins. T-cell proliferation was performed as described elsewhere and all assays were done in triplicates (24). Briefly, thawed PBMCs were seeded at $1.5 \times 10^{5}$ per well in 96-well plate in $250 \mu \mathrm{l}$ ex vivo 15 medium in the presence or absence of recombinant hTERT or survivin $(10 \mu \mathrm{g} / \mathrm{ml})$. After 6 days in culture, cells were pulsed with $\left[{ }^{3} \mathrm{H}\right]$-thymidine for $18 \mathrm{~h}$ prior to harvesting. An antigen-specific response was considered positive when the stimulatory index was more than three (24). Stimulation index was determined by dividing the mean counts per minute (cpm) of wells incubated with the antigen by the mean counts per minute of medium alone. To evaluate the strength of cell proliferation, the data are presented as cpm. Cytokine contents in culture supernatants were measured by commercially available ELISA kits (R\&D Systems).

Analysis of IDO expression by western blotting. IDO expression in the Fast DC preparations subsequent to siRNA transfection was analyzed using western blotting. The expression of IDO was also analyzed in mDCs generated using a standard method to produce monocyte-derived mDCs for clinical use $(18,19)$. In this protocol, monocytes obtained from leukapheresis were cultured for five days with GM-CSF and IL-4. Subsequently, they were cultured for two days with IL-1, IL-6, TNF- $\alpha$ and PGE2, and then frozen until use.

Flow cytometry. Phenotype of DCs was analysed by direct immunofluorescence staining of cell surface antigens using FITC or PE conjugated antibodies against CD80, CD83, CD86, HLA-DR, CCR7, CD40 and isotype controls. All antibodies were purchased from Dako (Glostrup, Denmark) or eBioscience (BD Biosciences, Pharmingen, San Diego, CA). After staining on ice for $30 \mathrm{~min}$, samples were washed twice and then analysed by flow cytometry using a FACSCanto II. The data were analysed using FlowJo software.

Analysis of tryptophan catabolism. To analyse tryptophan catabolism, dendritic cells were plated in Hanks' buffered salt solution (HBSS) containing $100 \mu \mathrm{ML}$-tryptophan (Sigma-Aldrich, St. Louis, MO, USA). Subsequent to overnight incubation at $37^{\circ} \mathrm{C}$, supernatants were harvested and analysed for tryptophan contents by commercially available ELISA kit (Abnova, Taipei City, Taiwan).

Statistical analysis. All in vitro assays were performed in triplicate, and values are plotted as mean \pm standard deviation. Values were compared using Student's t-test. P-values $<0.05$ were considered significant.

\section{Results}

IDO expression in standard DC vaccine preparations. Expression of IDO by DCs may create local tolerance by directly suppressing T-cell activation and enhancing regulatory T-cell function. Therefore, we have analysed IDO expression in clinical grade DCs generated for therapeutic vaccination of cancer patients. In total, 10 DC preparations were analysed by western blotting and all expressed IDO at the protein level (Fig. 1A as an illustrative example). These data are in accordance with recent reports showing IDO upregulation in human DCs upon in vitro maturation (25). The induction of IDO expression subsequent to in vitro maturation of DCs might be a major problem for the failure of DC-based immunotherapy.

IDO gene silencing in Fast DC vaccine preparations. Having demonstrated that IDO is expressed in DC vaccine preparations, next we analysed the silencing potency of the in vitro transcribed siRNAs. This siRNA was designed based on our previous study reporting on the identification of IDO siRNAs that function at low concentrations (16). In this study, siRNA-4 was optimized for efficient in vitro transcription under standard GMP conditions (18). Fast DC preparations from patients with ovarian cancer were transfected ex vivo with IDO siRNA along with mRNA encoding tumour antigens hTERT or survivin. As shown in Fig. 1B, the siRNA effectively inhibited IDO expression in all 8 fast DC vaccine preparations.

To ascertain the enzymatic activity of IDO, we have measured tryptophan catabolism in supernatants of IDO-silenced DCs and IDO-positive DCs using tryptophan 


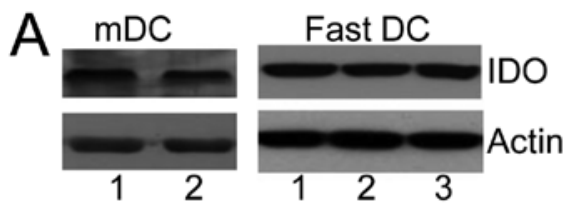

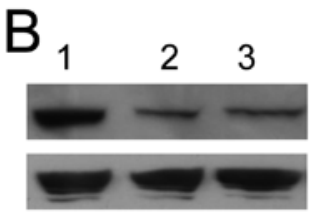

CU-1

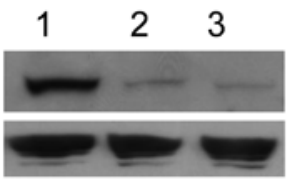

CU-2

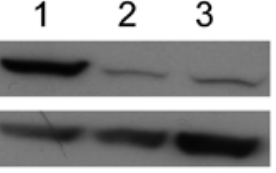

CU-3

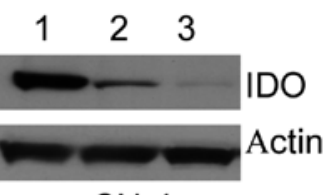

CU-4

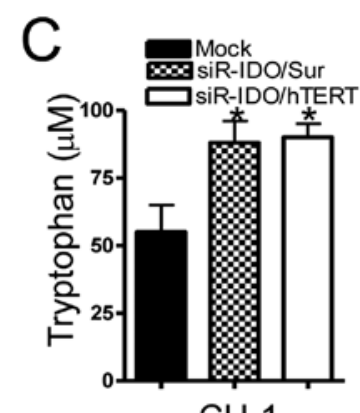

CU-1

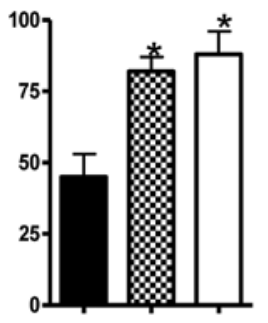

CU-2

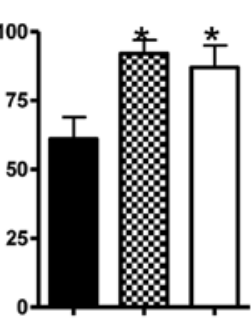

CU-3

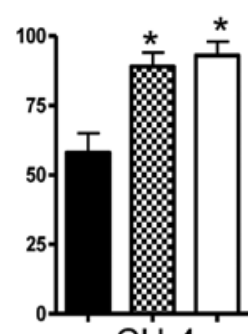

CU-4

Figure 1. IDO expression and gene silencing in fast DC vaccines. Autologous DCs were generated from monocytes isolated from leukapheresis. (A) IDO expression was analyzed by western blots either in mature (m) DCs (2 patients) generated using standard maturation protocol or in fast DCs (3 patients). (B) Gene silencing. Fast DC vaccine preparations from 4 patients (CU-1, CU-2, CU-3 and CU-4) were transfected with IDO siRNA under GMP conditions as described in Materials and methods. After $24 \mathrm{~h}$ post-transfection time, IDO expression was investigated by western blots and the remaining cells were cryopreserved into vaccine doses. Lanes 1, 2 and 3 correspond to mock DCs, DCs transfected with IDO siRNA and mRNA encoding for hTERT, DCs transfected with IDO siRNA and mRNA encoding for survivin, respectively. (C) Tryptophan catabolism by fast DC cancer vaccines. Cells were cultured in Hanks' buffered salt solution supplemented with $100 \mu \mathrm{M}$ tryptophan and incubated at $37^{\circ} \mathrm{C}$ for $24 \mathrm{~h}$. Subsequently, tryptophan contents in culture supernatants were determined by ELISA. Mock cells did not receive IDO siRNA. The data are representative for 4 independent experiments. ${ }^{*} \mathrm{P}<0.05$.

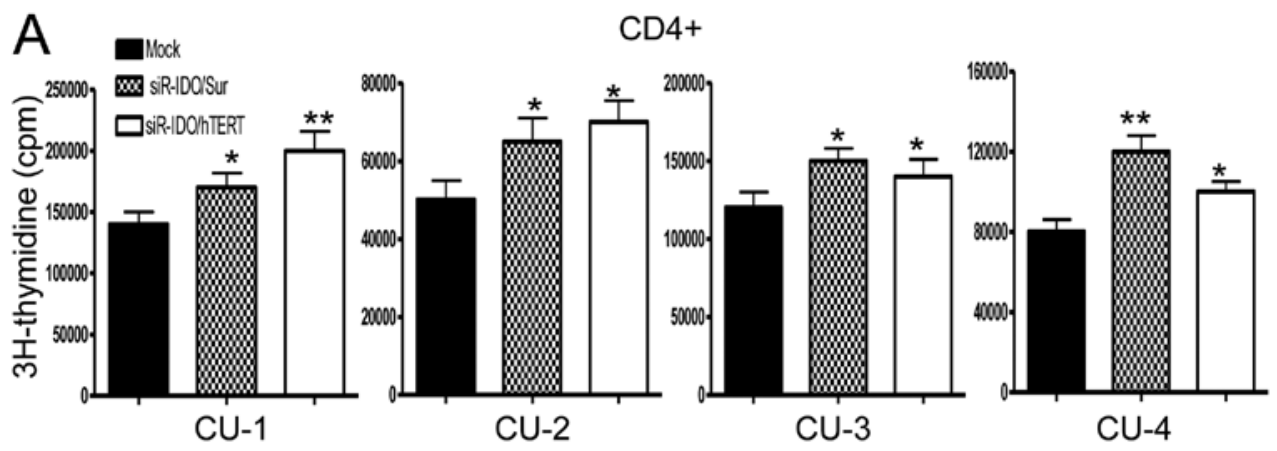

B

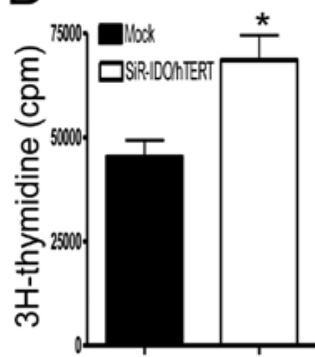

CU-1

CD8+

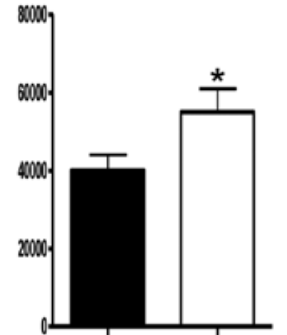

CU-2

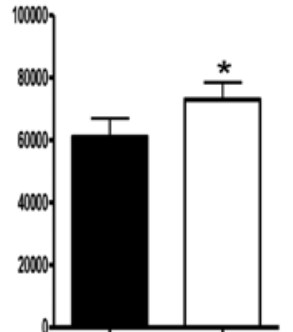

CU-3

Figure 2. Potency of IDO-silenced DC in stimulating allogeneic T cell proliferation. Patient IDO-silenced DCs and IDO-positive DC vaccine preparations were cocultured with either $\mathrm{CD}^{+}(\mathrm{A})$ or $\mathrm{CD}^{+}(\mathrm{B}) \mathrm{T}$ cells from a healthy donor at a T cell/DC ratio of $1: 5$. Cells were co-cultured for 5 days at $37^{\circ} \mathrm{C}$ and $\mathrm{T}$ cell proliferation was determined by incorporation of $\left[{ }^{3} \mathrm{H}\right]$-thymidine following overnight pulsing. The results are presented as mean \pm standard deviation of triplicate determinations. The data are representative for 3 independent experiments. ${ }^{*} \mathrm{P}<0.05,{ }^{* *} \mathrm{P}<0.02$. 


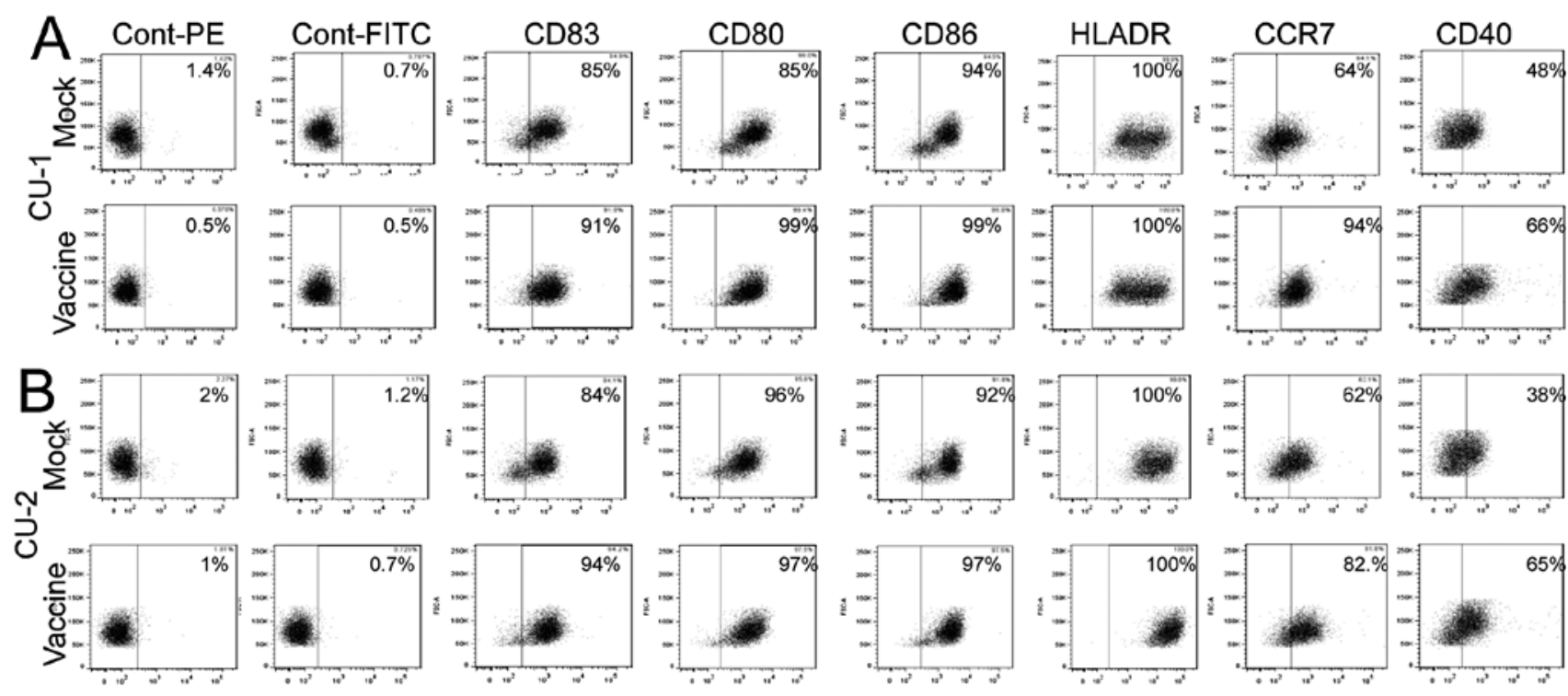

Figure 3. Phenotypic analysis of IDO-silenced DCs. Fast DC preparations from 2 patients (A and B) were transfected under GMP conditions with IDO siRNA and mRNA encoding hTERT tumour antigen (vaccine) and cryopreserved as described in Materials and methods. Mock cells did not receive IDO siRNA. Thawed DC vaccines and mock DCs were cultured overnight and then the expression of the indicated markers was analyzed by flow cytometry. The percentages of positive cells are shown.

ELISA kit. In contrast to IDO-silenced DCs, the tryptophan concentration decreased significantly in IDO-positive DCs (mock, $\mathrm{P}<0.05$ ) (Fig. 1C). Therefore, IDO is a functional enzyme in clinical DC preparations as suggested previously (25).

Allogeneic stimulatory potency of IDO-silenced DC vaccines. While DC-based therapy holds great promise for treating cancers, the clinical responses of conventional DC cancer vaccines are very low according to many studies (7). Furthermore, it is not yet known to what extent IDO contribute to tumour-related immune suppression in cancer patients. To determine if IDO-silenced DC activate T cells more efficiently than IDO-positive DCs, mixed lymphocyte reaction assay was used to measure the allostimulatory potency of these cells (Fig. 2A and B). IDO-silenced DC vaccine preparations showed a higher allostimulatory capacity of both $\mathrm{CD}^{+}$and $\mathrm{CD}^{+} \mathrm{T}$ cells than their counterparts IDO-positive DCs (mock DCs, $\mathrm{P}<0.05$ ), suggesting that the presence of tryptophan is a limiting factor in the cultures. The data are also consistent with the consumption of tryptophan by IDO-positive DCs shown in Fig. 1C.

Phenotypic analysis of DC vaccine preparations. The surface expression of various molecules related to antigen presentation, migration and T-cell activation was analysed by means of flow cytometry. The expression of CD80, CD83, CD86, and HLA-DR molecules was comparable in IDO-silenced DCs (vaccine) and IDO-positive DCs (mock) (Fig. 3A and B, as representative examples). However, in three patients (CU-1, CU-2 and CU-3), the expression of CD40 and CCR7 was significantly increased in IDO-silenced DCs when compared to their mock-transfected counterparts $(\mathrm{P}<0.05)$. For example, the percentages of IDO-silenced DC expressing CCR7 from patients CU-1, CU-2 and CU-3 were 94, 82 and 67\%, while that in mock DCs were 64, 62 and 47\%, respectively. CCR7 is required for DC migration to draining lymph nodes where they activate T cells. The upregulation of CCR7 and CD40 is most likely due to the activation of intracellular retinoid acid inducible-gene 1 (RIG-1) by 5'-triphosphate siRNA (see Discussion). It should be noted that the expression profiles of the analysed markers in others fast DC preparations transfected with mRNA encoding hTERT or survivin are similar to those of patient mock DCs (data not shown).

Immune and clinical responses. We next examined the safety of the DC vaccine and whether it would induce antigen-specific immune response and clinical improvement. The immunization was well tolerated except for the development of flu-like symptoms, which is reminiscent of effective induction of immunity. All four patients developed a DTH response (Table II). The induction of specific T-cell responses during treatment was analysed in peripheral blood before treatment, and at week 9-10 after vaccination. In this assay, we used recombinant hTERT and survivin proteins, enabling the detection of most T-cell epitopes. As shown in Fig. 4, the patients developed $\mathrm{T}$-cell response against hTERT and survivin tumour antigens. Notably, there was a 2- to 6-fold increase in T-cell proliferation when compared to unstimulated PBMCs.

To determine the nature of the immune response, we have assessed the levels of interferon $\gamma$ (IFN- $\gamma$ ), interleukin (IL)-4, IL-10 and IL-17 in T-cell culture supernatants from patients who responded to hTERT protein. A significant increase in IFN- $\gamma$ was detected in three patients, indicating a Th-1 type response (Fig. 5). IFN- $\gamma$ helps $\mathrm{CD}^{+} \mathrm{T}$ cells to differentiate into cytotoxic $\mathrm{T}$ lymphocytes capable of killing MHC class I positive tumours. 
Table II. Immune and clinical responses to IDO-DC vaccination.

\begin{tabular}{|c|c|c|c|c|}
\hline identity & DTH response & Immune-response & IDO-DCs (months) & IDO-DCs (months) \\
\hline CU-1 & + & + & 12 & 4.5 \\
\hline CU-2 & + & + & 10 & $13\left(+9^{a}\right)$ \\
\hline CU-3 & + & + & 0 & $21^{b}$ \\
\hline CU-4 & + & + & 14 & 11 \\
\hline
\end{tabular}

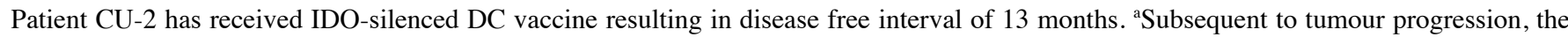
IDO-silenced DC vaccine was combined with chemotherapy. Patient CU-3 never had a tumour free interval in spite of multiple cycles of

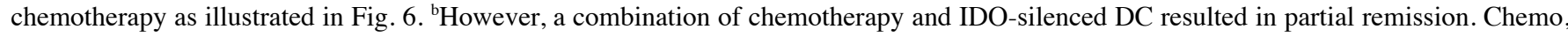
chemotherapy.
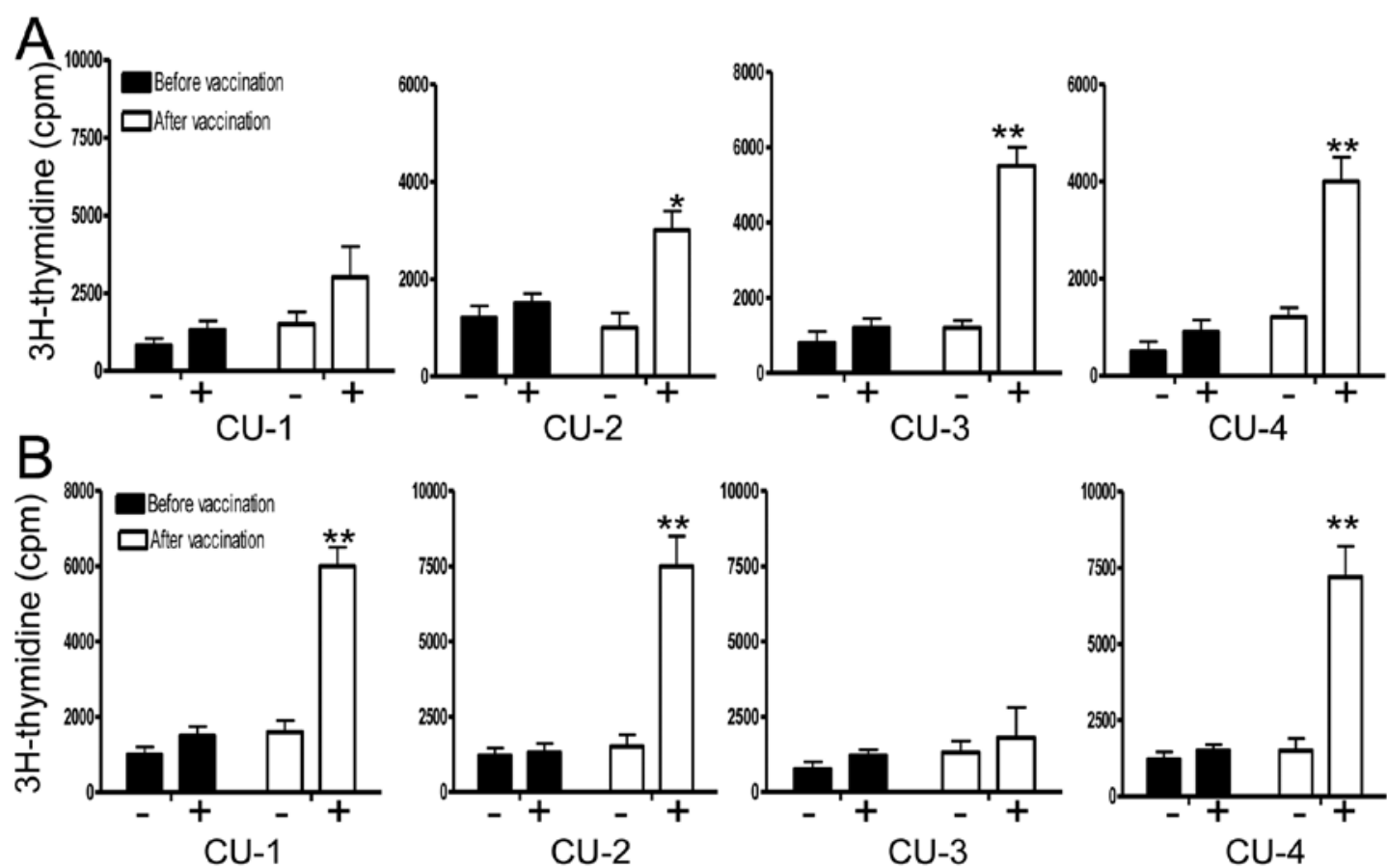

Figure 4. Analysis of T cell responses to hTERT and survivin tumour antigens. T cell responses to hTERT (A) and survivin (B) before start of vaccination and at week 9-10 after vaccination were analyzed in PBMCs. The cells $\left(1.5 \times 10^{5} / 250 \mu \mathrm{l}\right.$ medium) were incubated (+) or not (-) with recombinant hTERT or survivin protein $(10 \mu \mathrm{g} / \mathrm{ml})$ for 6 days and T-cell proliferation was determined by incorporation of $\left[{ }^{3} \mathrm{H}\right]$-thymidine following overnight pulsing. The results are presented as mean \pm standard deviation of triplicate determinations. The data are representative of 3 independent experiments. ${ }^{*} \mathrm{P}<0.05,{ }^{* *} \mathrm{P}<0.02$.

With regard to the clinical response (Table II), the four vaccinated patients maintained stable disease subsequent to vaccination. Interestingly, patient CU-2 and CU-3 showed a significant disease free interval compared to that obtained with only chemotherapy ( 13 vs 10 months, and 21 vs 0 months, respectively). When patients progressed following DC vaccination they were given a combination of both chemotherapy and DC vaccine (CU-2, CU-4). In patient $\mathrm{CU}-2$ this combination resulted in a partial remission and the patient is still under therapy. It should be noted that patient CU-3 never achieved a complete disease free interval on chemotherapy since 2007. However, when she was given DC vaccination combined with chemotherapy she obtained a partial remission and the decline in tumour volume and markers continues. This observation supports the potential benefits of combining DC vaccine and chemotherapy (26). Fig. 6 illustrates the treatment schedule and clinical outcome of patient CU-3.

\section{Discussion}

The clinical efficacy of DC-based vaccines for cancer immunotherapy is influenced by various interplaying, immunomodulating factors including IL-10 and IDO. In the present study, we evaluated the safety and efficacy of an IDO-silenced DC vaccine in four patients with ovarian cancers extensively pre-treated with standard chemotherapy and with recurrent disease. We show that even in these patients, it is possible to 

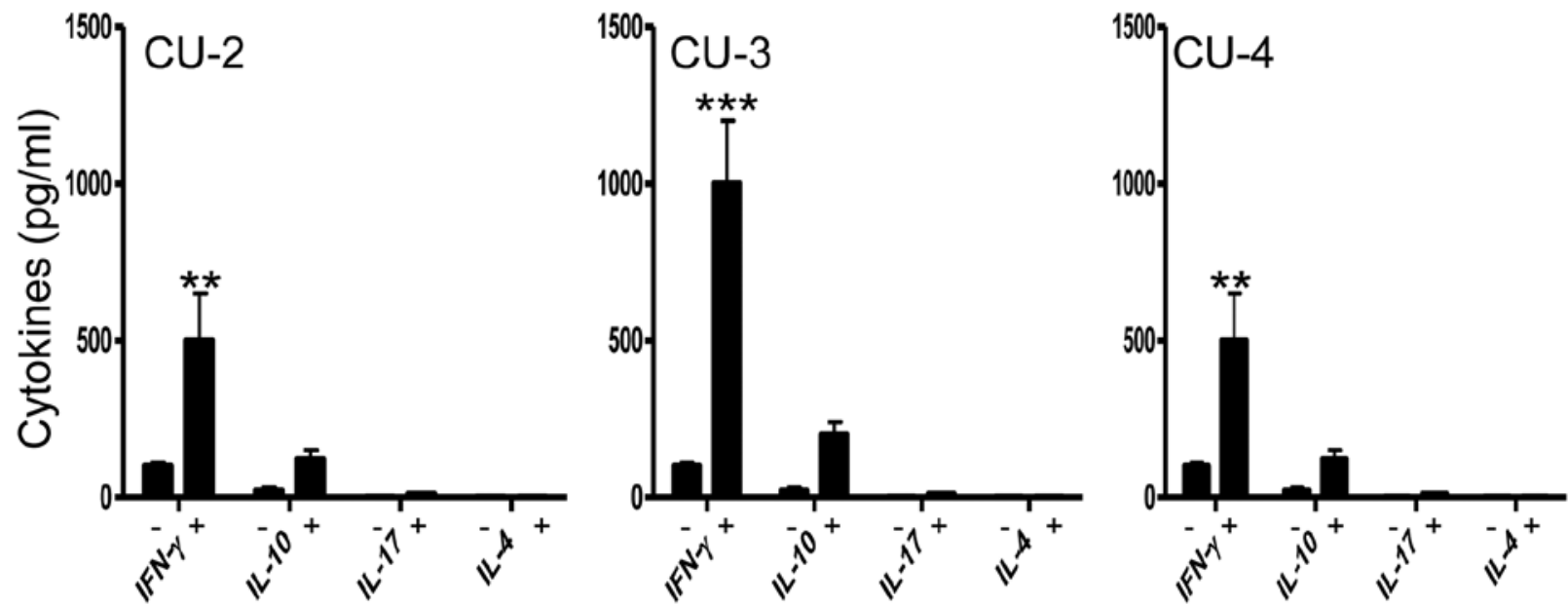

Figure 5. Cytokine contents in culture supernatants. PBMCs were stimulated (+) or not (-) with recombinant hTERT (10 $\mu \mathrm{g} / \mathrm{ml})$ for 4 days and then cytokine contents in culture supernatants were analyzed by commercially available ELISA kits. The results are presented as mean \pm standard deviation of triplicate determinations. The data are representative of 4 independent experiments. ${ }^{* *} \mathrm{P}<0.02,{ }^{* * *} \mathrm{P}<0.002$.

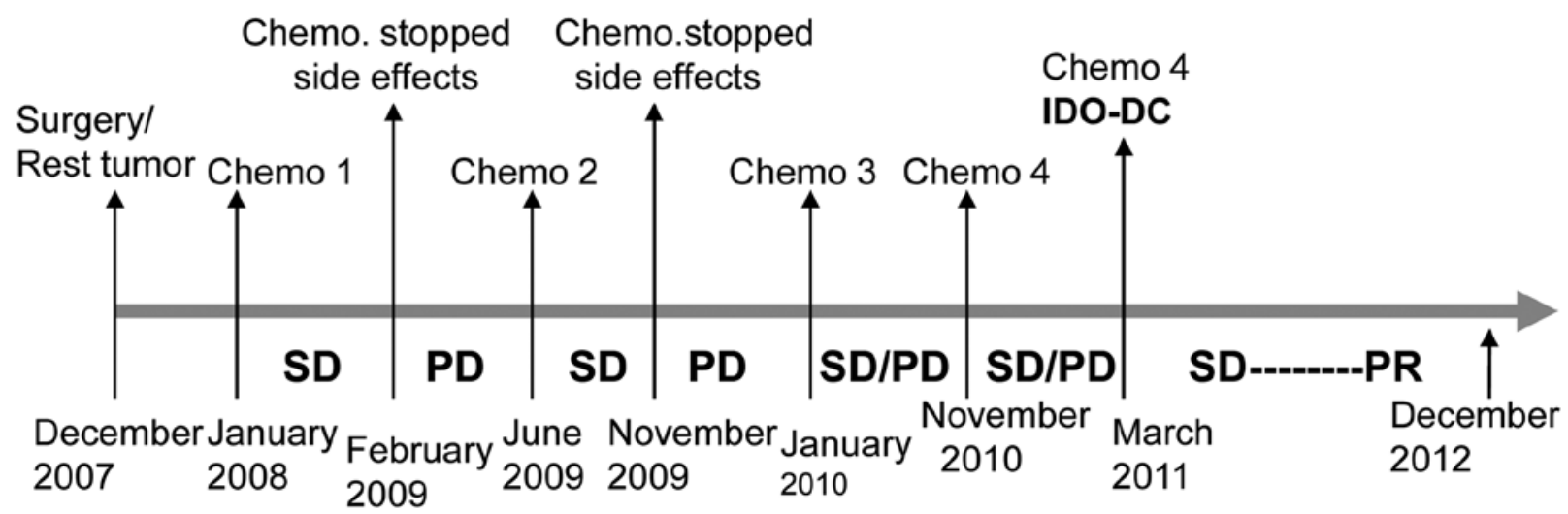

Figure 6. Timeline for the treatment and clinical development. The figure illustrates the treatment schedule for the patient with ovarian cancer (CU-3). After surgery, the patient has received four combinations of chemotherapy (chemo) prior DC vaccine. Chemo 1, carboplatin, taxol, avastin; chemo 2, carboplatin, caleyx; chemo 3, taxol; chemo 4, taxol, avastin; SD, stable disease; PD, progressive disease; PR, partial remission.

induce immune responses leading to clinical improvement. Indeed, all four patients developed a DTH response and flu-like symptoms after vaccination, and a strong hTERTspecific T-cell response was detected in peripheral blood of three patients. Also, the patients developed survivin-specific $\mathrm{T}$-cell response. These immune responses were related to objective clinical responses. Given these encouraging findings, the downregulation of IDO expression in DC cancer vaccines should be considered as a new attractive approach for generating therapeutic immunity against tumours.

In general, the development of an immune response is controlled by DCs (1). Once T cells are effectively primed, pro-inflammatory cytokines such as IFN- $\gamma$ and T-reg signals such as CTLA-4, induce IDO expression in DCs resulting in their conversion into tolerogenic DCs. Such DCs can inhibit T-cell expansion as well as the generation of adaptive T-reg cells from naïve $\mathrm{CD}^{+} \mathrm{T}$ cells $(27)$. IFN $-\gamma$ is produced primary by NK cells, activated $\mathrm{CD} 4^{+}$and $\mathrm{CD} 8^{+} \mathrm{T}$ cells and NKT cells Reverse signalling via B7 molecules (CD80/86) expressed on DCs after interaction with CD28 on T cells has also been shown to induce IDO expression in DCs. With respect to cancer vaccines, the induction of IDO expression can occur during in vitro maturation of DCs as well as in vivo after T-cell activation as indicated above. IDO gene silencing by siRNA is expected to block IDO expression during this entire process and thereby enhancing the potency of DC vaccine. Gene silencing in fast DCs did not weaken after nine days in culture (data not shown). The inclusion of siRNAs into DC cancer vaccines is a straightforward strategy that does not require additional technology or modification to the mRNA transfection procedure (18). Moreover, the data suggest that RNAi using IDO siRNA is safe in humans. Indeed, none of the vaccinated patients have developed other side-effects than flu-like symptoms following vaccination. Flu-like symptoms are considered to be a good sign of efficient immune responses (7). It should be noted that vaccination of mice with suppressor of cytokine signalling-1 (SOCS-1)-silenced DCs also enhanced antigen-specific antitumor immunity in in vivo murine models (28). This provides more evidence to support the knockdown of immuno-suppressive genes in DC 
cancer vaccines. In this respect, a vaccine that combines the inhibition of IDO, IL-10 and SOCS-1 is under investigation.

Out of the four vaccinated patients, only one patient (CU-2) is HLA-A2 positive. However, pentamer staining with a newly identified hTERT T-cell epitope (674-683 pentamer) 19 did not detect any peripheral CTL response, indicating that the response to recombinant hTERT was directed towards other T-cell epitopes that are under investigation. Patient PBMC incubated with hTERT protein produced a significant amount of IFN- $\gamma$, but not IL-4 suggesting the induction of Th-1 immune polarization that may be contributed to the clinical responses seen in the patients.

Another feature of the IDO-silenced DC vaccine is the upregulation of CD40 and CCR7 seen in 3 out of 4 vaccinated patients. Previously, we have shown that in vitro transcribed siRNA carrying uncapped 5'-triphosphate can activate immature monocyte-derived DCs via intracellular pathogen recognition receptor RIG-1 $(16,29)$. RIG-1 is a cytosolic sensor of viral RNA-bearing 5'-triphosphate, triggering a type 1-interferon and proinflammatory cytokine response $(30,31)$. A recent study also showed that in vitro transcribed siRNAs targeting Bcl-2 induced the expression of interferons and activated DCs in a mouse model when injected intravenously (32). Similarly, Kubler et al (33) showed that RIG-1 activation can trigger cytokine secretion and DC maturation. The ability of siRNA to activate cytolytic RIG-1 in DCs coupled with the silencing of gene expression raises the prospect of 5'-triphosphate-bearing siRNAs being developed as therapeutics and adjuvant for cancer vaccines. Simulating viral infection with agents such as uncapped 5'-triphosphate siRNA, rather than aseptic inflammation (using cytokine cocktails) can induce the differentiation of monocytes into DCs with optimal function. In this respect, Cheong et al demonstrated that blood monocytes can be mobilized to develop into 'real' DCs in vivo by microbial danger signals such as lipopolysaccharide (34). They acquired antigen-presenting function equal to or in some cases superior to bone marrow derived DCs.

The discovery of RNAi has revolutioned molecular biology by allowing selective knockdown of genes in a manner more potent than that of any other previous strategies (35). Despite growing interest in the use of siRNA in humans (36-39), one of the major challenges to the clinical development of gene silencing by siRNAs has been in vivo delivery in general, and particularly the ability to selectively target siRNA molecules to specific cell types $(40,41)$. The use of siRNA in an ex vivo setting as demonstrated in the current study should overcome the delivery and targeting problems mentioned above. With respect to cancer immunotherapy, our data also indicate that transfection with IDO siRNA does not have a negative effect on DC phenotype and function. In contrast, it does produce 'superior' DCs compared to their mock transfected counterparts. The in vitro enhanced DC function seen may contribute to the clinical improvement seen in patients following vaccination. These encouraging results, coupled with recent findings that siRNA-bearing 5'-triphosphate can activate DCs, should be further explored as a platform for inducing enhanced antitumor immunity in humans. The current study sets the stage for testing IDO-silenced DC vaccine in cancer patients. Existing DC-based vaccine clinical trials would likely benefit from additional modifications to silence inhibitory molecules such as IDO, IL-10 and SOCS-1.

\section{Acknowledgements}

The authors are grateful for assistance provided by the Cell therapy members for vaccine preparation and clinical follow-up of the patients. This study was supported in part by the Norwegian Ministry of Health, the Norwegian Cancer Society and Inger and John Fredriksen Foundation for ovarian Cancer.

\section{References}

1. Steinman RM and Banchereau J: Taking dendritic cells into medicine. Nature 449: 419-426, 2007.

2. Banchereau J and Steinman RM: Dendritic cells and the control of immunity. Nature 392: 245-252, 1998.

3. Jego G, Pascual V, Palucka AK and Banchereau J: Dendritic cells control B cell growth and differentiation. Curr Dir Autoimmun 8: 124-139, 2005.

4. Shaw J, Wang YH, Ito T, Arima K and Liu YJ: Plasmacytoid dendritic cells regulate B-cell growth and differentiation via CD70. Blood 115: 3051-3057, 2010.

5. Steinman RM, Hawiger D and Nussenzweig MC: Tolerogenic dendritic cells. Annu Rev Immunol 21: 685-711, 2003.

6. Hoos A, Britten CM, Huber C and O'Donnell-Tormey J: A methodological framework to enhance the clinical success of cancer immunotherapy. Nature Biotechnol 29: 867-870, 2011.

7. Palucka $\mathrm{K}$ and Banchereau J: Cancer immunotherapy via dendritic cells. Nature Rev 12: 265-277, 2012.

8. Sioud M and Sørensen D: Generation of an effective anti-tumor immunity after immunization with xenogeneic antigens. Eur J Immunol 33: 38-45, 2003.

9. Sioud M: Does our current understanding of immune tolerance, autoimmunity, and immunosuppressive mechanisms facilitate the design of efficient cancer vaccines? Scand J Immunol 70: 516-525, 2009.

10. Munn DH, Sharma MD, Lee JR, Jhaver KG, Johnson TS, Keskin DB, Marshall B, Chandler P, et al: Potential regulatory function of human dendritic cells expressing indoleamine 2,3-dioxyggenase. Science 297: 1867-1870, 2002.

11. Hwu P, Du MX, Lapointe R, Do M, Taylor MW and Young HA: Indoleamine 2,3-dioxygenase production by human dendritic cells results in the inhibition of $\mathrm{T}$ cell prolifieration. $\mathrm{J}$ Immunol 164: 3596-3599, 2000

12. Braun D, Longman RS and Albert ML: A two-step induction of indoleamine 2,3 dioxygenase (IDO) activity during dendritic cell maturation. Blood 106: 2375-2381, 2005.

13. Munn DH, Sharma MD and Hou D: Expression of indoleamine 2,3-dioxygenase by plasmacytoid dendritic cells in drainingdraining lymph noedes. J Clin Invest 114: 280-290, 2004.

14. Jonuleit H, Schmitt E, Stenbrink K and Enk AH: Dendritic cells as a tool to induce anergic and regulatory $\mathrm{T}$ cells. Trends Immunol 22: 394-400, 2001.

15. Sørensen RB, Berge-Hansen L, Junker N, Hansen CA, Hadrup SR, Schumacher TN, Svane IM, Becker JC, thor Straten P and Andersen MH: The immune system strikes back: cellular immune responses against indoleamine 2,3-dioxygenase. PLoS One 4: e6910, 2010.

16. Flatekval GF and Sioud M: Modulation of dendritic cell function and maturation with mono- and bifunctional small interfering RNAs targeting indoleamine 2,3-dioxygenase. Immunology 128: e837-e848, 2010.

17. Guppy AE, Nathan PD and Rustin GJ: Epithelial ovarian cancer: a review of current management. Clin Oncol 17: 399-411, 2005.

18. Sæbøe-Larssen S, Fossberg E and Gaudernack G: mRNA-based electrotransfection of human dendritic cells and induction of cytotoxic T lymphocyte responses against the telomerase catalytic subunit (hTERT). J Immunol Methods 259: 191-203, 2002 .

19. Suso EMI, Dueland S, Rasmussen AM, Vetrhus T, Aamdal S, Kvalheim G and Gaudernack G: hTERT mRNA dendritic cell vaccination: complete response in a pancreatic cancer patient associated with response against several hTERT epitopes. Cancer Immunol Immunother 60: 809-818, 2011.

20. Tanaka F, Yamaguchi H, Haraguchi N, Mashino K, Ohta M, Inoue $\mathrm{H}$ and Mori $\mathrm{M}$ : Efficient induction of specific cytotoxic $\mathrm{T}$ lymphocytes to tumor rejection peptide using functional matured 2 day-cultured dendritic cells derived from human monocytes. Int J Oncol 29: 1263-1268, 2006. 
21. Liu JP, Chen W, Schwarer AP and Li H: Telomerase in cancer immunotherapy. Biochim Biophys Acta 1805: 35-42, 2010.

22. Andersen MH, Svane IM, Becker JC and Straten PT: The universal character of the tumor-associated antigen survivin. Clin Cancer Res 13: 5991-5994, 2007.

23. Moberslein $M$ and Sioud M: Galectin-1 and -3 gene silencing in immature and mature dendritic cells enhances T cell activation and interferon- $\gamma$ production. J Leuko Biol 91: 461-467, 2012.

24. Sioud M, Kjeldsen-Kragh J, Quayle AJ, Wiker HG, Sørskaar D, Natvig JB and Førre O: Immune responses to 18.6 and $30-\mathrm{kDa}$ mycobacterial antigens in rheumatoid patients, and $\mathrm{V}$ beta usage by specific synovial T-cell lines and fresh T cells. Scand J Immunol 34: 803-812, 1991.

25. Wobser M, Voigt $\mathrm{H}$ and Houben R: Dendritic cell based antitumor vaccination: impact of functional indoleamine 2,3-dioxygenase expression. Cancer Immunol Immunother 56: 1017-1024, 2007.

26. Hodge JW, Ardiani A, Farsaci B, Kwilas AR and Gmeiro SR The tiping point for combination therapy: cancer vaccines with radiation, chemotherapy, or targeted small molecule inhibitors. Semin Oncol 39: 323-339, 2012.

27. Munn DH and Mellor AL: IDO expression by dendritic cells: tolerance and tryptophan catabolism. Nat Rev Immunol 4 762-774, 2004

28. Shen L, Evel-Kabler K, Strube R and Chen SY: Silencing of SOCS1 enhances antigen presentation by dendritic cells and antigen-specific anti-tumor immunity. Nat Biotechnol 22 1546-1553, 2004.

29. Furset $G$ and Sioud M: Design of bifunctional siRNAs: combining immunostimulation and gene-silencing in one single siRNA molecule. Biochem Biophys Res Commun 352: 642-649, 2007.

30. Hornung V, Ellegast J, Kim S, Brzózka K, Jung A, Kato H, Poeck H, et al: 5'-Triphosphate RNA is the ligand for RIG-I. Science 314: 994-997, 2006.

31. Pichlmair A, Schulz O, Tan CP, Naslund TI, Liljestrom P, Weber F and Reis e Sousa C: RIG-I-mediated antiviral responses to single-stranded RNA bearing 5'-phosphates. Science 314: 997-1001, 2006.
32. Poeck H, Besch R and Maihoefer C: 5'-triphosphate-siRNA: turning gene silencing and RIG-I activation against melanoma. Nat Med 14: 1256-1263, 2008.

33. Kubler K, Gehrke N and Riemann S: Targeted activation of RNA helicase retinoic acid-inducible gene-I induces proimmunogenic apoptosis of human ovarian cancer cells. Cancer Res 70: 5293-5304, 2012.

34. Cheong C, Matos I and Choi JH: Microbial stimulation fully differentiates monocytes to DC-SIGN/CD209(+) dendritic cells for immune T cell areas. Cell 143: 416-429, 2010.

35. Fire $\mathrm{A}, \mathrm{Xu} \mathrm{S}$ and Montgomery MK: Potent and specific genetic interference by double-stranded RNA in Caenorhabditis elegans. Nature 391: 806-811, 1998.

36. Kaiser PK, Symons RCA and Shah SM: A randomized, double-blind, placebo-controlled study of an RNAi-based therapy directed against respiratory syncytial virus. Proc Natl Acad Sci USA 107: 8800-8805, 2010.

37. Zamora MR, Budev M and Rolfe M: RNA interference therapy in lung transplant patients infected with respiratory syncytial virus. Am J Resp Critical Care Med 183: 531-538, 2011.

38. DiGiusto DL, Krishnan A and Li L: RNA-based gene therapy for HIV with lentiviral vector-modified CD34(+) cells in patients undergoing transplantation for AIDS-related lymphoma. Sci Transl Med 2: 36ra43, 2010.

39. Leachman SA, Hickerson, RP and Schwartz ME: First-inhuman mutation-targeted siRNA Phase I trial of an inherited skin disorder. Mol Ther 18: 442-446, 2010.

40. Tiemann K and Rossi JJ: RNAi-based therapeutics-current status, challenges and prospects. EMBO Mol Med 1: 142-151, 2009.

41. Behlke MA: Progress towards in vivo use of siRNAs. Mol Ther 13: 644-670, 2006. 